\title{
Overcoming polar-format issues in synthetic aperture radar multichannel autofocus
}

\author{
Hyun Jeong Cho $\bowtie$, David C. Munson, Jr. \\ Department of Electrical Engineering and Computer Science, University of Michigan, Ann Arbor, MI, USA \\ 凶-mail: zzon@umich.edu
}

\begin{abstract}
Multichannel autofocus (MCA) is a subspace-based autofocus method for solving the defocusing problem in synthetic aperture radar. In addition to the one-dimensional (1D) defocusing assumption, MCA assumes that the perfectly focused image has a low-return region, which is naturally guaranteed by the spatially limited nature of the radar antenna footprint. In theory, MCA yields better or even perfect solutions compared to other autofocus methods. However, the authors have discovered that MCA is far more sensitive to violation of the 1D defocusing assumption compared to other methods; in fact, MCA is unsuitable for even fairly small data-collection angles. Fortunately, this problem can be solved if they reverse the order of two steps in the image formation process and apply MCA in a domain where the defocusing effect is one dimensional. The distorted version of the image, obtained by inverse Fourier transforming the polar-grid data without further interpolation, contains regions satisfying a low-return assumption, but the region of low return must be carefully specified for best performance. They present simulation results of the proposed method, reversed-step MCA, for various ranges of look angles and discuss the selection of lowreturn constraints.
\end{abstract}

\section{Introduction}

Synthetic aperture radar (SAR) is a microwave imaging system that achieves a narrow effective beam, and thus high cross-range resolution, by synthetically increasing the size of the aperture. In the tomographic formulation of spotlight-mode SAR, the demodulated data corresponding to a vantage angle $\theta$ are essentially a slice of the two-dimensional (2D) Fourier transform of the reflectivity at angle $\theta[1]$. Thus, SAR data lies on a polar grid in the Fourier domain, and a natural means of image formation is to apply $2 \mathrm{D}$ inverse Fourier transformation after interpolating the data onto a Cartesian grid.

Perfect demodulation requires accurate knowledge of the distance between the centre of the image patch and the radar platform for each vantage angle. Although modern GPS-equipped SAR systems can estimate the distance with fair accuracy, for high frequency SARs, it is difficult to measure the distance within a small fraction of the wavelength from a moving platform, as needed. As a result, the demodulated data typically are contaminated with unknown phase shifts. When imaging from space, unknown phase shifts also can be imparted by spatially-varying and time-varying properties of the ionosphere. In the presence of the phase errors, the resulting image is improperly focused [2].

In recent decades, a number of solutions to the defocusing problem, referred to as autofocus methods, have been proposed and studied [3-8]. Most autofocus algorithms are well-motivated, but heuristic. A more recently proposed autofocus method, named multichannel autofocus (MCA) is a subspace-based approach that directly solves for the correction filter [8]. MCA assumes that a small portion of the perfectly focused image is zero-valued, or at least nearly zero-valued, which is nearly satisfied in practice due to the spatial limit of the antenna beam. Under the image support constraint, in principle, MCA can provide a better or even perfect restoration of the focused image, compared to other methods. Furthermore, unlike most autofocus methods, the performance of MCA does not depend heavily on the nature of the phase error.
MCA and other efficient autofocus algorithms estimate the phase errors based on the assumption that the effect of defocusing on the image is $1 \mathrm{D}$. This assumption is nearly satisfied when the range of data collection angles is small, so that the polar raster for the acquired Fourier data does not deviate much from a Cartesian grid. However, as the range of angles becomes wider, the blurring kernel no longer can be approximated as a 1D function in the cross-range direction, and so the algorithms fail to focus the image properly. Although many SAR applications use a narrow range of data collection angles (a few degrees), there are applications, such as bistatic SAR imaging, that require a wider range of data collection angles (up to tens of degrees).

We showed in [9] that MCA is particularly sensitive to the 1D defocusing assumption and proposed to improve the MCA approach by interchanging the interpolation step and the autofocus step; we applied MCA to the corrupted inverse-polar data, which is obtained by inverse Fourier transforming the polar-grid data without interpolation. By interchanging the steps, the defocusing effect is $1 \mathrm{D}$, and thus we can obtain better estimates of the phase errors using the subspace-based technique. However, we must account for how the low-return region changes in the inverse-polar data. By using modified low-return constraints, this method, which we refer to as reversed-step MCA (RMCA), gives near-perfect restoration in many cases. This paper gives a complete and updated account of the preliminary work reported in [9].

The organisation of this paper is as follows. Section 2 presents the SAR autofocus problem and establishes the notation used in this paper. The reversed-step reconstruction and the modified low-return constraints are studied in Section 3. For analytical purposes, we consider a continuous version of the inverse-polar domain and study point target responses. The analysis provides a guideline for determining the low-return constraint for the RMCA reconstruction. In Section 4, simulation results are presented for different ranges of look angles and different choices of low-return constraints. Some practical issues also are discussed. We conclude in Section 5. 


\section{Problem statement and MCA}

\subsection{SAR defocusing problem}

In our development, we consider spotlight-mode SAR because this high-resolution form of SAR ordinarily uses short wavelengths, where the need for autofocus is most pressing.

The geometry of an airborne SAR imaging scenario is shown in Fig. 1. The radar platform illuminates the terrain patch and collects the backscattered pulses, which are acquired for look angles $\theta_{l}$ : $\theta_{\min } \leq \theta_{l} \leq \theta_{\max }, l=0,1, \ldots, M-1$, where $\Theta=\theta_{\max }-\theta_{\min }$ is the range of look angles. The patch to be imaged is depicted as the shaded circular region of diameter $L$, and the radar is at a distance $R_{\theta}$ from the centre of the scene when the look angle is $\theta$. The direction of the flight path is referred to as the cross-range or azimuth direction and the direction perpendicular to the flight path is referred to as the range direction. Here, the goal is to reconstruct the complex-valued reflectivity $q(x, y)$ of the ground patch.

Returns from each look angle are demodulated using knowledge of the round-trip delay $2 R_{\theta} / c$. Under the assumption that the radar is operating in the far field, i.e. when $R_{\theta} \gg L$, the demodulated return from look angle $\theta$ is a slice of the 2D Fourier transform $Q$ $\left(f_{X}, f_{Y}\right)$ of $q(x, y)$, taken at an angle $\theta$ [1]. In practice, due to the limited and tapered support of the radar antenna beam, the demodulated returns correspond to the weighted reflectivity

$$
g(x, y)=q(x, y) w(x, y),
$$

where $w(x, y)$ is the antenna beam pattern, whose support is nearly limited to a particular region in space. Then discrete samples of the demodulated data from angle $\theta_{l}$ are described by

$$
G_{\mathrm{p}}[l, k]=G\left(f_{\mathrm{R}}[k] \cos \theta_{l}, f_{\mathrm{R}}[k] \sin \theta_{l}\right), \quad k=0, \ldots, N-1,
$$

where $G\left(f_{X}, f_{Y}\right)$ is the $2 \mathrm{D}$ Fourier transform of $g(x, y)$, and $\left\{f_{\mathrm{R}}[k]\right\}$ are the radial samples of the demodulated data. The region of support of the acquired Fourier data is an annulus, offset from the origin, with the radial extent determined by the waveform carrier frequency and bandwidth, and angular extent that is the same as the range of data collection angles [1].

Since the SAR data are samples of the 2D Fourier transform of $g$, a natural means of image formation uses 2D inverse Fourier transformation. To utilise fast algorithms for Fourier transformation, the polar-grid data usually is first interpolated onto a Cartesian grid. The interpolated SAR data can be described by

$$
G_{\mathrm{c}}\left[l^{\prime}, k^{\prime}\right]=\sum_{l=0}^{M-1} \sum_{k=0}^{N-1} G_{\mathrm{p}}[l, k] \Psi_{\mathrm{PC}}\left[l^{\prime}, k^{\prime} ; l, k\right],
$$

where $\Psi_{\mathrm{PC}}$ is a polar-to-Cartesian interpolation kernel, and $l^{\prime}$ and $k^{\prime}$ are indices corresponding to sample points in the range and cross-range spatial frequency, respectively. By taking a $2 \mathrm{D}$ inverse discrete Fourier transform (DFT) of $G_{\mathrm{c}}$, we obtain the image $g_{\mathrm{c}}[m, n]=\mathrm{DFT}_{l^{\prime}, k^{\prime}}^{-1}\left\{G_{\mathrm{c}}\left[l^{\prime}, k^{\prime}\right]\right\}$.

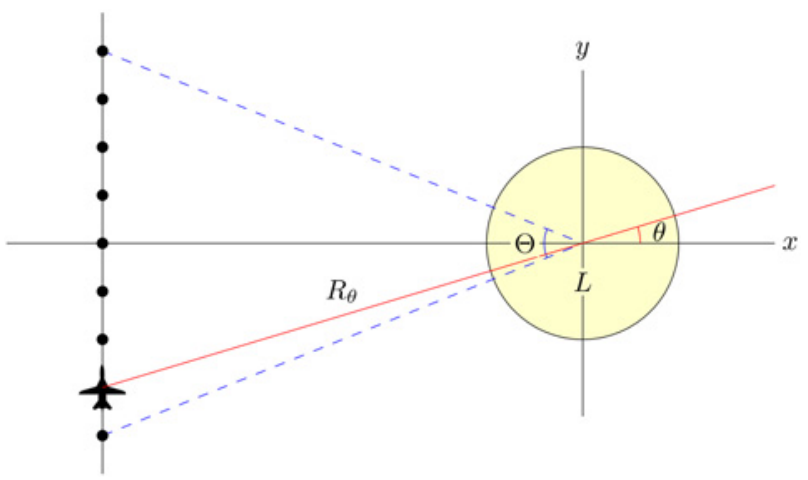

Fig. 1 Geometry of data collection in spotlight-mode SAR
An accurate reconstruction of the image $g_{c}$ requires precise knowledge of any variation in the round-trip distances, $2 R_{\theta}$, which may be difficult to measure within a small fraction of a wavelength. As a result, each of the demodulated returns is contaminated with an unknown phase shift; the contaminated demodulated returns can be described by

$$
\tilde{G}_{\mathrm{p}}[l, k]=G_{\mathrm{p}}[l, k] \mathrm{e}^{\mathrm{j} \phi_{l}}, \quad \forall l, k,
$$

where $\phi_{l}$ is the unknown phase shift corresponding to the return from look angle $\theta_{l}$ [2]. Generally, the measurement errors are different for each return, and the resulting phase errors are considered to be a 1D function of the look angle. In the presence of phase errors, the reconstructed image becomes improperly focused, and the resulting challenge is referred to as the SAR defocusing problem.

\section{$2.21 D$ autofocus methods}

The aim of SAR autofocus is to restore the perfectly focused image $g_{\text {c }}$ given the set of corrupted SAR data $\left\{\tilde{G}_{\mathrm{p}}[l, k]\right\}$ and assumptions about the characteristics of the underlying scene. A common assumption is that the range of look angles $\Theta$ is small, so that the polar data grid in the Fourier domain is nearly Cartesian. Then, the corrupted data can be approximated as

$$
\tilde{G}_{\mathrm{c}}\left[l^{\prime}, k^{\prime}\right]=G_{\mathrm{c}}\left[l^{\prime}, k^{\prime}\right] \mathrm{e}^{\mathrm{j} \phi_{1 D}\left[l^{\prime}\right]}, \quad \forall l^{\prime}, k^{\prime},
$$

where $\phi_{1 \mathrm{D}}$ is a $1 \mathrm{D}$ phase-error function depending only on the cross-range index $l^{\prime}$. Since the resulting image under this model is blurred only in one spatial direction, the small-angle assumption is also known as the $1 D$ defocusing assumption.

Existing efficient autofocus methods estimate the phase-error function $\phi_{1 \mathrm{D}}$ under the small-angle assumption and other reasonable assumptions. For example, phase gradient autofocus (PGA) [3] implicitly assumes certain characteristics of the imaging scene, such as strong reflectors in the various range bins; and a statistical model is assumed in [4], providing the basis for maximum-likelihood estimation. Metric-based autofocus methods [5-7] assume the suitability of a particular image focus metric, such as image entropy $[5,7]$. Entropy of an image $\boldsymbol{g}$ is defined as

$$
E(\boldsymbol{g}) \triangleq-\sum_{m, n}|\bar{g}[m, n]|^{2} \ln \left(|\bar{g}[m, n]|^{2}\right),
$$

where

$$
\bar{g}[m, n] \triangleq g[m, n] /\left(\sum_{m, n}|g[m, n]|^{2}\right)
$$

is the power-normalised image. Lower entropy generally corresponds to better focus, and we will use image entropy as a performance measure of autofocus algorithms throughout this paper.

\subsection{Multichannel autofocus}

MCA is a direct approach to the defocusing problem, which assumes that a small portion of the perfectly-focused image is zero-valued, or least nearly zero-valued [8]. This assumption of a low-return region is satisfied in practice due to the spatial limitation of the antenna footprint and oversampling.

Like most autofocus methods, MCA was developed under the small-angle approximation. In matrix notation, MCA describes the autofocused image $\hat{\boldsymbol{g}} \in \mathbb{C}^{N_{Y} \times N_{X}}$ in terms of the corrupted Cartesian-grid Fourier data $\tilde{\boldsymbol{G}}_{\mathrm{c}} \in \mathbb{C}^{N_{Y} \times N_{X}}$ and phase compensations $\hat{\phi} \in \mathbb{C}^{N_{Y}}$ as

$$
\hat{g}[m, n]=\boldsymbol{\Phi}_{\mathrm{MCA},\{[m, n]\}} \mathbf{e}^{-\mathrm{j} \hat{\phi}}, \quad \forall m, n
$$

where $\boldsymbol{e}^{-\mathrm{j} \hat{\phi}}$ is an $N_{Y} \times 1$ vector with entries 
$\mathrm{e}^{-\mathrm{j} \hat{\phi}_{l}}, l=0, \ldots, N_{Y}-1$, and $\boldsymbol{\Phi}_{\mathrm{MCA},\{[m, n]\}}$ is a $1 \times N_{Y}$ vector with its $\left(l^{\prime}+1\right)$ th entry being

$$
\Phi_{\mathrm{MCA},\{[m, n]\}}^{\left(l^{\prime}\right)}=\sum_{k^{\prime}=0}^{N_{X}-1} \tilde{G}_{\mathrm{c}}\left[l^{\prime}, k^{\prime}\right] \exp \left\{\mathrm{j} 2 \pi\left(\frac{n k^{\prime}}{N_{X}}+\frac{m l^{\prime}}{N_{Y}}\right)\right\} .
$$

(Note that this formula is different from the one in the original paper [8]; however, they are equivalent descriptions.)

Now, let $\Omega \subset\left[0: N_{Y}-1\right] \times\left[0: N_{X}-1\right]$ denote the set of discrete indices corresponding to the presumed low-return region, i.e.

$$
|\hat{g}[m, n]| \simeq 0, \quad \forall[m, n] \in \Omega .
$$

The samples of the phase-compensated image that correspond to the presumed low-return region can be concatenated into a vector: $\hat{\boldsymbol{g}}_{\Omega}=\boldsymbol{\Phi}_{\mathrm{MCA}, \Omega} \mathbf{e}^{-\mathrm{j} \phi}$, where $\boldsymbol{\Phi}_{\mathrm{MCA}, \Omega}$ is a $|\Omega| \times M$ matrix, composed of row vectors $\left\{\boldsymbol{\Phi}_{\mathrm{MCA}_{,}\{[m, n]\}}:[m, n] \in \Omega\right\}$. MCA aims to find the phase compensations $\hat{\phi}$ that minimise $\left\|\hat{\boldsymbol{g}}_{\Omega}\right\|_{2}$ and estimates the phase errors by

$$
\hat{\phi}_{\mathrm{MCA}}=-\angle\left\{\underset{\|v\|_{2}=1}{\arg \min }\left\|\boldsymbol{\Phi}_{\mathrm{MCA}, \Omega} \boldsymbol{v}\right\|_{2}\right\} .
$$

When the presumed low returns are exactly zero and $\boldsymbol{\Phi}_{\mathrm{MCA}, \Omega}$ has rank $N_{Y}-1$, MCA recovers the focused image exactly. However, the restoration is not guaranteed to be exact in most other cases. The accuracy of the estimate is greatly related to the choice of low-return pixels and their values; thus, the low-return constraints must be chosen carefully.

\section{Reversed-step MCA}

\subsection{MCA and the polar-format issue}

MCA is based on the 1D defocusing model described by (5). In reality, the actual Fourier data lies on a polar grid and the corrupted Cartesian-grid data are described as

$$
\tilde{G}_{\mathrm{c}}\left[l^{\prime}, k^{\prime}\right]=\sum_{l=0}^{M-1} \sum_{k=0}^{N-1} G_{\mathrm{p}}[l, k] \Psi_{\mathrm{PC}}\left[l^{\prime}, k^{\prime} ; l, k\right] \mathrm{e}^{\mathrm{j} \phi[l]},
$$

and are not related to the uncorrupted data $G_{\mathrm{c}}\left[l^{\prime}, k^{\prime}\right]$ by multiplicative $1 \mathrm{D}$ phase errors. Although MCA is based on an erroneous model, the amount of deviation from the 1D defocusing model has been assumed to be small when the polar data grid is nearly Cartesian, i.e. when the range of look angles $\Theta$ is small, so that the effect on the performance of MCA would be correspondingly small. However, the modelling error can grow very fast as the polar grid deviates from a Cartesian grid, and so the performance of MCA can be much poorer than expected.

It is difficult to construct a simple model for the 2D phase errors in $\tilde{G}_{\mathrm{c}}$; however, in [9], we compared the dependence of the performance of $\mathrm{MCA}$, on the departure from a $1 \mathrm{D}$ phase error, with that of two other 1D autofocus methods: PGA and minimum entropy autofocus. By applying an additive independent 2D random phase error uniformly distributed between $-\sigma \pi$ and $\sigma \pi$, all three algorithms showed degradation in performance as the amount of deviation $\sigma$ became larger. Moreover, while MCA exhibited outstanding performance when the defocusing effect was truly 1D, it degraded the most rapidly as $\sigma$ became larger. A possible reason for the high sensitivity of MCA to the departure from the 1D defocusing model is that the individual singular vectors of a matrix may be unstable, i.e. small perturbations in data can result in a considerably different set of singular vectors [10].

Even if the phase errors in $\tilde{G}_{\mathrm{c}}$ are truly $1 \mathrm{D}$, the error in the magnitude of $\tilde{G}_{\mathrm{c}}$ may also affect the performance of MCA. The role of Fourier magnitude in image reconstruction is known to be much less important than that of the phase [11], and a similar observation has been made for SAR data [12]. However, an error at a single Fourier sample point affects the entire image [13], and uncorrelated errors in Fourier magnitude may introduce uncorrelated errors in the reconstructed image, resulting in a loss of contrast [14]. Thus, the errors in the Fourier magnitude affect the region of low return, which is especially problematic when the contrast of the original scene is low, resulting in poor performance of MCA. Although not presented in this paper, simulation results showed that the amount of magnitude error in the interpolated Fourier data is large when the phase errors are uncorrelated, and, as expected, the average energy in the presumed low-return region increased as the range of look angles became wider. However, the amount of degradation in the performance of MCA due to the magnitude errors was much smaller than that caused by the $2 \mathrm{D}$ phase errors.

\subsection{Basic idea}

Hence MCA suffers from inaccuracy of the 1D phase assumption in the Cartesian domain, we propose estimating the phase errors before applying polar-to-Cartesian interpolation. For a given polar data grid in the 2D Fourier space, we define the inverse-polar data $\boldsymbol{g}_{\mathrm{p}}$ of an image $g_{a}$ as

$$
g_{\mathrm{p}}[m, n]=\sum_{l=0}^{M-1} \sum_{k=0}^{N-1} G_{\mathrm{p}}[l, k] \mathrm{e}^{\mathrm{j} 2 \pi((m l / M)+(n k / N))},
$$

where $\left\{\boldsymbol{G}_{\mathrm{p}}\right\}$ is the Fourier transformation of $g_{a}$ collected on a polar grid. We call the space of inverse-polar data the inverse-polar domain. (Note that we use this more accurate terminology rather than 'warped-domain data' and 'warped domain' as in our preliminary work [9].)

Clearly, the inverse-polar data are corrupted by a 1D blurring kernel, regardless of the range of look angles. Thus, we can expect better performance in estimating the blurring kernel, so long as we can identify an appropriate low-return region in the inverse-polar domain. Then, we can correct the errors in $\left\{\tilde{\boldsymbol{G}}_{\mathrm{p}}\right\}$ and obtain the autofocused image by the usual polar format algorithm (polar-to-Cartesian interpolation followed by a $2 \mathrm{D}$ fast Fourier transform). We call our method RMCA since the order of image formation and autofocus are reversed.

\subsection{Impulse response in the inverse-polar domain}

Since a low-return region is naturally provided by the antenna footprint in practice, the low-return constraints for MCA are straightforward to choose. The low-return region in the inverse-polar domain also depends on the shape of the antenna footprint, but in a less straightforward way. To gain insight into what the inverse-polar data might look like, we derive impulse responses for a continuous-version of the inverse-polar domain. Although we make assumptions in deriving the formula, for the tractability of our analysis, the result will help us understand where the low-return regions are in the inverse-polar domain.

Instead of having a discrete set of Fourier samples $\left\{G_{\mathrm{p}}[l, k]\right\}$, assume that we have access to the Fourier data $G_{\mathrm{p}}\left(f_{\mathrm{R}}, \theta\right)=G$ $\left(f_{\mathrm{R}} \cos \theta, f_{\mathrm{R}} \sin \theta\right)$ for $f_{\mathrm{R}} \in\left[f_{0}-\Delta_{f} / 2, f_{0}+\Delta_{f} / 2\right]$ and $\theta \in[-\Theta / 2, \Theta / 2]$. The continuous inverse-polar data $g_{\mathrm{p}}\left(x_{\mathrm{p}}, y_{\mathrm{p}}\right)$ can be defined as

$$
g_{\mathrm{p}}\left(x_{\mathrm{p}}, y_{\mathrm{p}}\right) \triangleq \iint G_{\mathrm{p}}\left(f_{\mathrm{R}}, \theta\right) \mathrm{e}^{i 2 \pi\left(x_{\mathrm{p}} f_{\mathrm{R}}+f_{0} y_{\mathrm{p}} \theta\right)} \mathrm{d} f_{\mathrm{R}} \mathrm{d} \theta
$$

Suppose we have an impulse at location $\left(r_{0} \cos \theta_{0}, r_{0} \sin \theta_{0}\right)$ in the spatial domain, where we assume that $r_{0}>0$. Then, the inverse-polar data corresponding to the impulse is

$$
h_{\mathrm{p}}\left(x_{\mathrm{p}}, y_{\mathrm{p}}\right)=\Delta_{f} \mathrm{e}^{i 2 \pi f_{0} x_{\mathrm{p}}} \int_{-\Theta / 2}^{\Theta / 2} A\left(x_{\mathrm{p}}, \theta\right) \mathrm{e}^{-i \omega\left(y_{\mathrm{p}}, \theta\right)} \mathrm{d} \theta,
$$



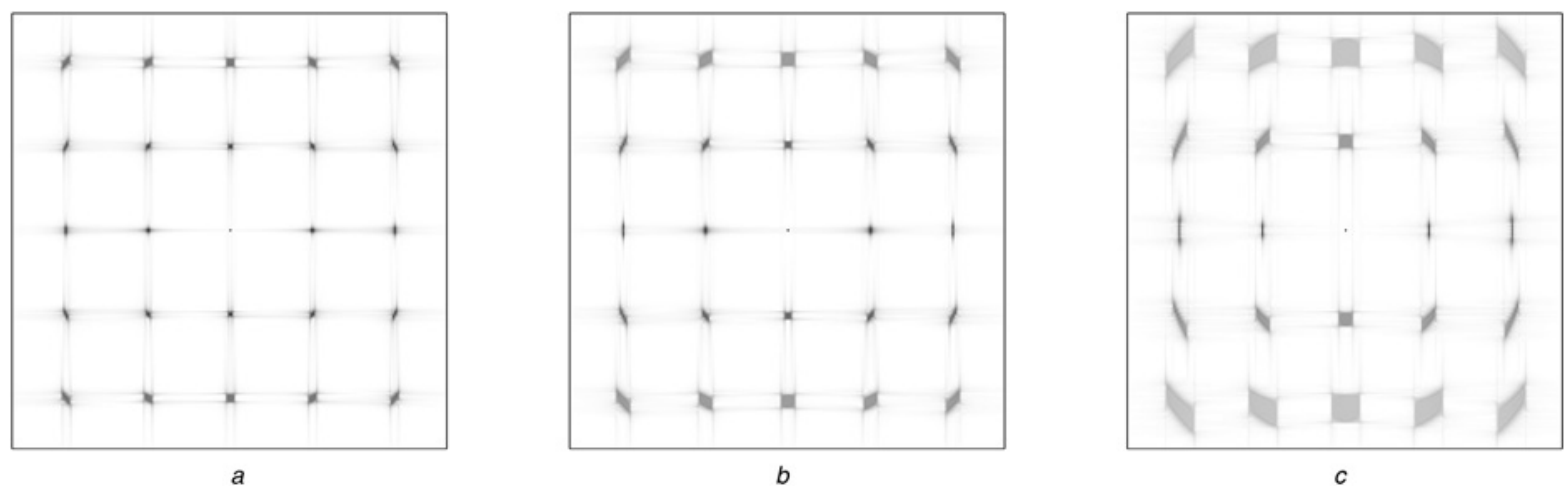

Fig. 2 Inverse-polar data corresponding to a scene consisting of 25 point reflectors

$a$ For $\Theta=3^{\circ}$

$b$ For $\Theta=5^{\circ}$

$c$ For $\Theta=10^{\circ}$

Amplitude is plotted on a logarithmic scale

where

$$
A\left(x_{\mathrm{p}}, \theta\right)=\operatorname{sinc}\left[\Delta_{f}\left\{x_{\mathrm{p}}-r_{0} \cos \left(\theta_{0}-\theta\right)\right\}\right]
$$

and

$$
\omega\left(y_{\mathrm{p}}, \theta\right)=2 \pi f_{0}\left\{r_{0} \cos \left(\theta_{0}-\theta\right)-y_{\mathrm{p}} \theta\right\} .
$$

The centre frequency, $f_{0}$, is high in practice, and it follows that $\omega$ is rapidly varying as a function of $\theta$, whereas $A$ is relatively slowly varying. By the principle of stationary phase, $\left|h_{\mathrm{p}}\right|$ will have relatively large values if stationary phase is realised within the range of integration [15]. For a fixed $y$-coordinate $y_{\mathrm{p}}$, stationary phase is realised at $\theta^{*}\left(y_{\mathrm{p}}\right)=\theta_{0}-\arcsin \left(y_{\mathrm{p}} / r_{0}\right)$. If $\theta^{*}\left(y_{\mathrm{p}}\right) \in[-\Theta / 2$, $\Theta / 2]$, i.e. if

$$
y_{\mathrm{p}} \in\left\{r_{0} \sin \left(\theta_{0}-\theta\right): \theta \in\left[-\frac{\Theta}{2}, \frac{\Theta}{2}\right]\right\}
$$

then (14) can be approximated as

$$
h_{\mathrm{p}}\left(x_{\mathrm{p}}, y_{\mathrm{p}}\right) \simeq \frac{\Delta_{f} A\left(x_{\mathrm{p}}, \theta^{*}\left(y_{\mathrm{p}}\right)\right) \mathrm{e}^{i\left\{2 \pi f_{0} x_{\mathrm{p}}-\omega\left(y_{\mathrm{p}}, \theta^{*}\left(y_{\mathrm{p}}\right)\right)\right\}}}{\sqrt{-\mathrm{j} f_{0} r_{0} \cos \left\{\theta_{0}-\theta^{*}\left(y_{\mathrm{p}}\right)\right\}}}
$$

by using a second-order Taylor-series expansion of $\omega$ about the stationary point. From the approximation, we can see that $\left|h_{\mathrm{p}}\right|$ will have large values near the $x$-coordinate $x_{\mathrm{p}}=r_{0} \cos \left\{\theta_{0}-\theta^{*}\left(y_{\mathrm{p}}\right)\right\}$ for a fixed $y_{\mathrm{p}}$. Having assumed that the stationary phase point is within the range of integration, the impulse response in the inverse-polar domain will have large values near an arc described by

$$
\left(r_{0} \cos \theta, r_{0} \sin \theta\right), \quad \theta \in\left[\theta_{0}-\frac{\Theta}{2}, \theta_{0}+\frac{\Theta}{2}\right] .
$$

Note that the arc becomes longer as the range of look angles and distance of the point target from the centre of scene increase.

Fig. 2 shows simulated inverse-polar data for a scene consisting of 25 point reflectors of equal reflectivity and equal spacing in both directions. The simulation results agree with the above analysis, which was done for the continuous-version of the inverse-polar domain, showing impulse responses having arc-like features. Also, the sharp responses are smoothed as the range of look angles becomes wider and as distance from the origin of the scene increase, as suggested by the analysis.

The arc shapes of the impulse responses suggest a region of low return in the inverse-polar domain; the outermost corners of the inverse-polar domain are likely to have low returns, where the exact shape of the corner regions depends on the antenna pattern and the range of look angles. To test this hypothesis, we applied ideal circular and rectangular antenna patterns to a scene of scatterers of uniform strength and random phase, and then calculated Fourier data on a polar grid [12]. We then calculated inverse-polar data, averaged over multiple realisations of spatial phases to reduce the speckle.

Fig. 3 shows the synthesised inverse-polar data and their contours for ideal circular and rectangular antenna patterns with no sidelobes. Here, we took an average over 100 realisations for $\Theta=30^{\circ}$. For larger $\Theta$, the shape of the contours deviates more from the original window shape and expand outwards in the horizontal direction towards the edges.

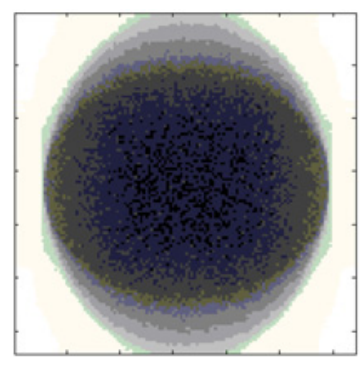

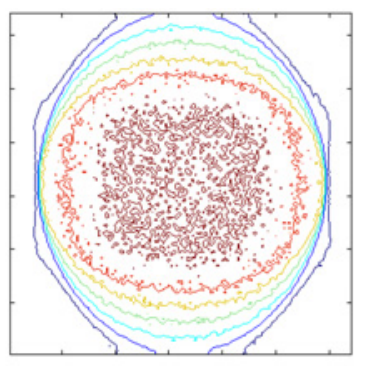

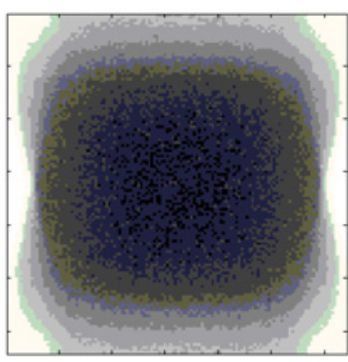

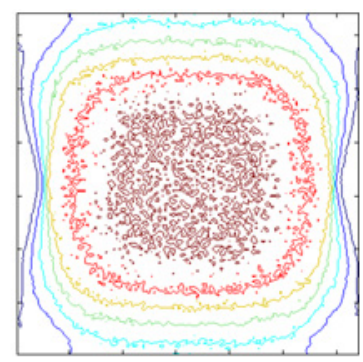

Fig. 3 Inverse-polar data corresponding to different antenna patterns for $\Theta=30^{\circ}$ : a inverse-polar data corresponding to a circular antenna footprint, $b$ contours of $a, c$ inverse-polar data corresponding to a rectangular antenna footprint, and $d$ contours of $c$ 


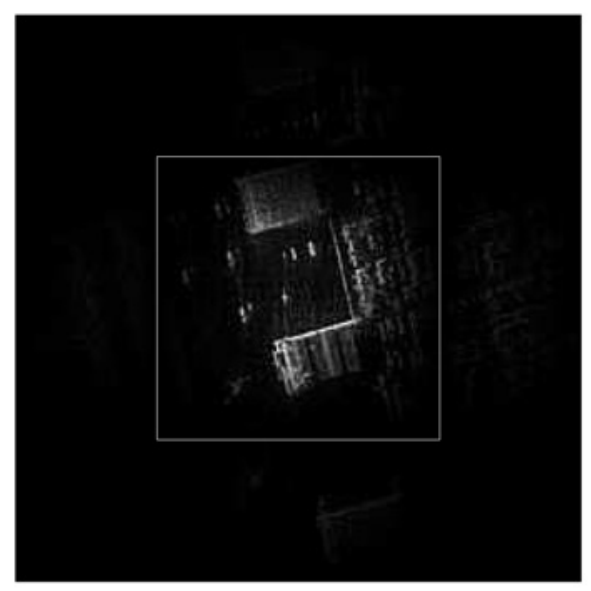

a

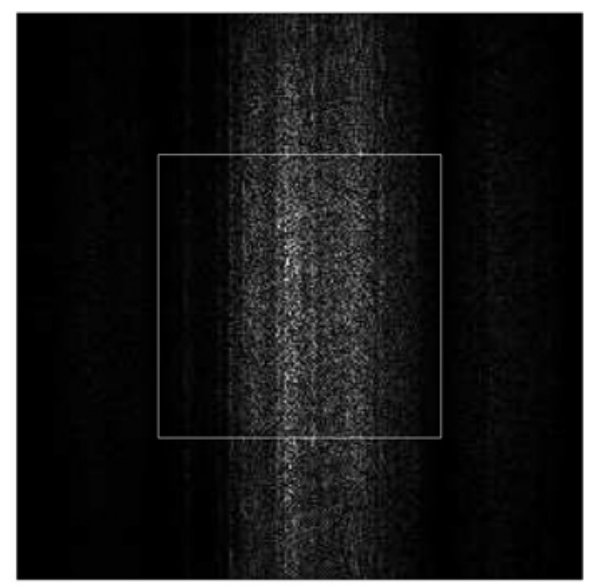

b

Fig. 4 Realistic simulation of SAR imaging. a SAR scene modelled by a $256 \times 256$ image with a $2 D$ separable sinc window applied, and $b$ image defocused by a white phase error function. The squares in $a$ and $b$ indicate the reduced FOV corresponding to the region of interest

\section{Simulation results}

\subsection{Simulation with $2 D$ separable sinc antenna footprint}

Fig. $4 a$ shows a realistic SAR scene, which was obtained by applying an antenna pattern to a SAR image made available from

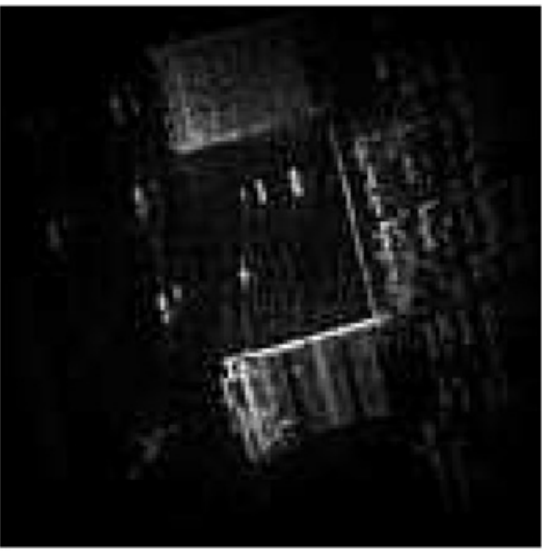

a

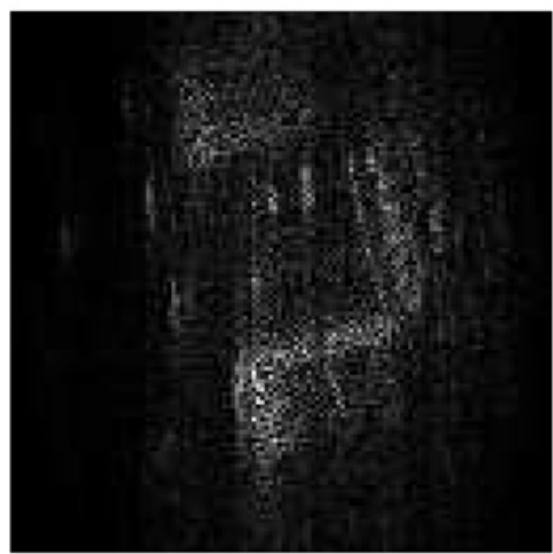

Sandia National Laboratories [16]. A 2D separable sinc function was used to model the attenuation by the antenna, where the main lobe of each sinc function covered half the width of the scene. The white square in the figure circumscribes the main lobe of the sinc, and later will be used as the borderline of a reduced field of view (FOV) for the restored images. Spatial phases were created

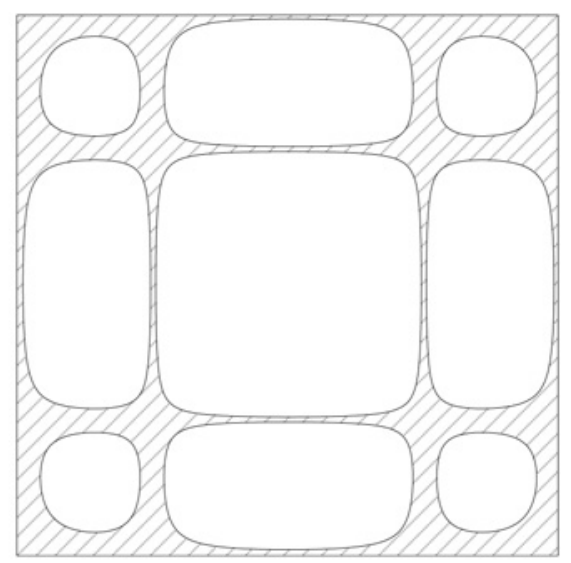

b

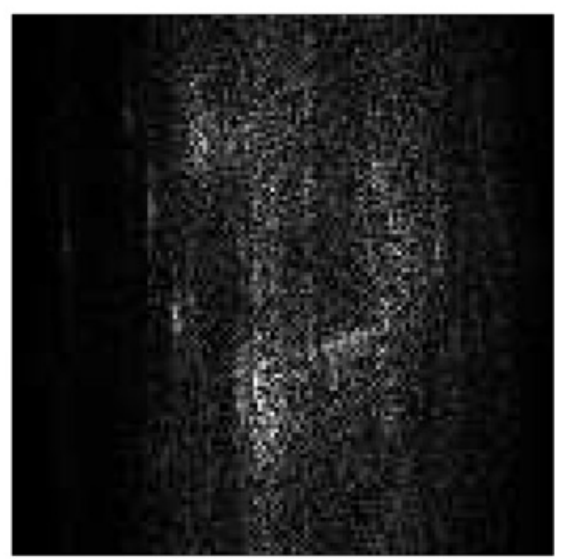

d

Fig. 5 Simulation of MCA. a Perfectly-focused image of size $128 \times 128$, formed in the absence of phase errors, $b$ presumed low-return region in the original FOV, $c$ $M C A$ restoration with the lowest entropy measure for $\Theta=0.1^{\circ}$, and $d M C A$ restoration with the lowest entropymeasure for $\Theta=1^{\circ}$. a, c, and d only show the restored image within the reduced FOV. Entropy of the images: a 7.6028, c 8.2562, and d 8.5056. The number of low-return constraints for MCA restorations: c 5376, and d 2048 
independently from a uniform distribution ranging from $-\pi$ to $\pi$. SAR data were then synthesised on a polar grid in the Fourier domain, and a white phase error function was applied to model a severe defocusing effect. We used PFA for SAR image formation, with the resolution and FOV being equal to that of the scene shown in Fig. $4 a$. Fig. $4 b$ shows a defocused image for $\Theta=0.1^{\circ}$. Images in Fig. 4 and the contours of the attenuation windows to be shown later are presented with a wide FOV in order to show the low-return region. However, we will use the reduced FOV for restored images since we generally are interested in imaging the region that is strongly illuminated.

A perfectly focused image for $\Theta=0.1^{\circ}$ is shown in Fig. $5 a$ for comparison purpose. For MCA, pixels corresponding to the lowest values of the antenna footprint were selected to comprise the low-return region, and the number of constraints was linearly increased from the number of cross-range indices in the defocused image to 24 times that minimum number. Among the restored images, the one with the lowest entropy measure was selected as the 'best' MCA restoration. The shaded region in Fig. $5 b$ shows the presumed low-return region that yielded the 'best' MCA restoration for $\Theta=0.1^{\circ}$, and Fig. 5c shows the corresponding MCA restoration. MCA works to some extent in this challenging scenario and we can see some features in this image; however, it is not nearly as sharp as the perfectly-focused image even though the polar data grid is nearly Cartesian. As discussed earlier, the restoration quality of MCA drastically decreases as the range of look angles becomes wider. This is illustrated in Fig. $5 d$ where the MCA restoration for $\Theta=1^{\circ}$ is poorly focused even though the data collection angle is still narrow. Restorations for $\Theta$ larger than about $3^{\circ}$ are not distinguishable from the defocused image.

To specify the low-return region for RMCA, attenuation windows corresponding to the antenna pattern were computed in the inverse-polar domain as described in the second last paragraph of Section 3.3. Again, RMCA was applied multiple times with the number of constraints ranging from the number of angular samples to 24 times the minimum number, and the image with the lowest entropy measure was selected as the 'best' RMCA restoration. The contours of the inverse-polar domain attenuation windows are shown in Figs. $6 a$ and $c$ for $\Theta=0.1^{\circ}$ and $\Theta=5^{\circ}$, respectively. They each show contours of the antenna footprint at three levels, each corresponding to different sets of low-return constraints; the three correspond to the low-return region with (i) the fewest number of constraints, (ii) the 'best' RMCA restoration, and (iii) the largest number of constraints. For $\Theta=0.1^{\circ}$, the shapes of contours in the inverse-polar domain are very close to those in the spatial domain. Accordingly, the low-return constraints for RMCA remain similar to the spatial-domain low-return constraints for MCA, yet, RMCA performs much better than MCA and produces a nearly perfect image, as shown in Fig. $6 b$. The improvement in quality is due to applying autofocus in the inverse-polar domain where the defocus truly is $1 \mathrm{D}$. As the range of look angles becomes wider, the inverse-polar domain attenuation windows become less similar to a 2D separable sinc function. Furthermore, the low-return pixel values of the attenuation windows become higher. Consequently, RMCA restorations are less focused for larger values of $\Theta$, although the degradation in RMCA performance is not nearly so severe as for MCA. Fig. $6 d$ shows
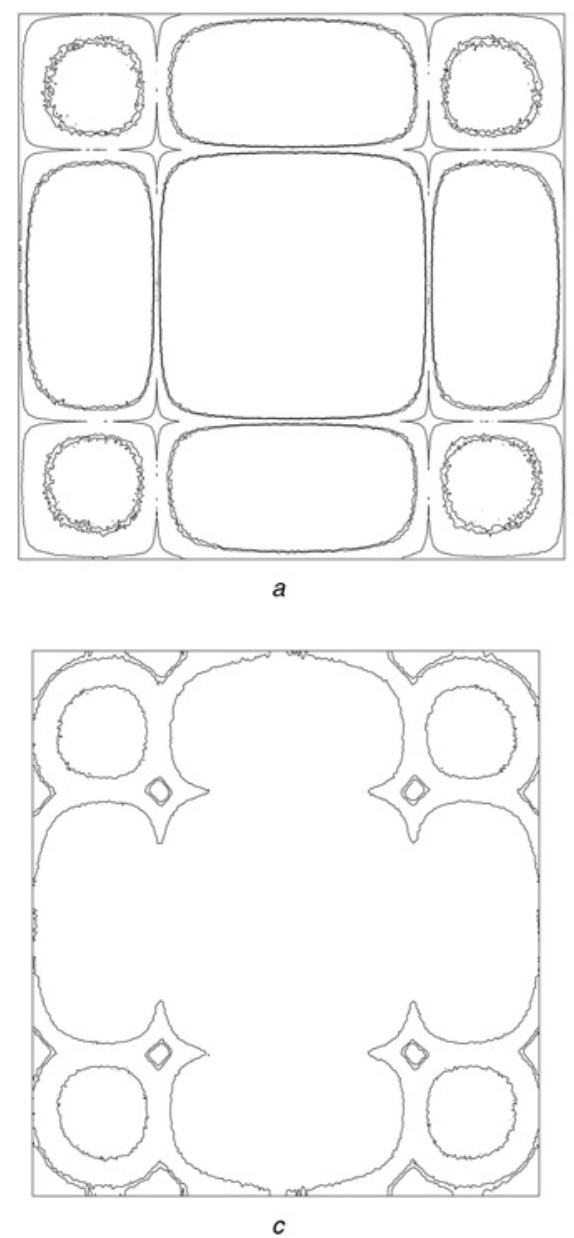

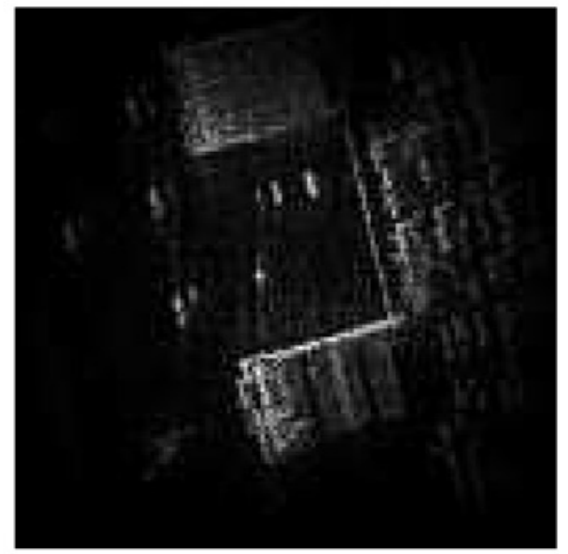

$b$

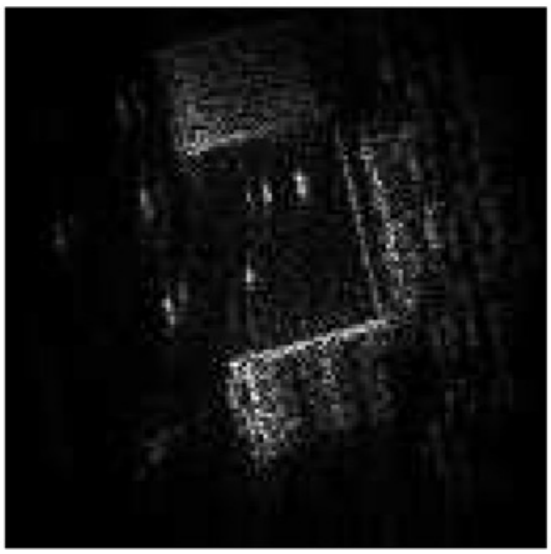

d

Fig. 6 Simulation of RMCA. a and c show contours of the inverse-polar domain attenuation window. The three contour levels of each figure correspond to three different sets of low-return constraints for RMCA. $b$ and $d$ are RMCA restorations with the lowest entropy measure. Entropy of the images: $b$ 7.5708 and $d 7.6335$. The number of low-return constraints for RMCA restorations: $b 5160$ and $d 840$ 
the RMCA restoration for $\Theta=5^{\circ}$, which still clearly shows most of the detail in the scene. RMCA successfully restores reasonably well-focused images even for fairly wide angles of $10^{\circ}-20^{\circ}$, and the extremely challenging white phase error used in these simulations. To conserve space, these results are not presented in this paper.

Fig. 7 shows how the performance of the multichannel autofocus algorithms relates to the range of look angles and the number of low-return constraints. The $x$ 's and crosses represent MCA for $\Theta=$ $0.1^{\circ}$ and $\Theta=1^{\circ}$, respectively, and the dots, stars, and pentagrams represent RMCA for $\Theta=0.1^{\circ}, \Theta=1^{\circ}$, and $\Theta=5^{\circ}$, respectively. Among the different sets of constraints, the ones corresponding to the lowest image entropy are marked with circles for each set of simulations. The performance is measured by image entropy and the image corresponding to (number of constraints) $=0$ is the defocused image. Regardless of the number of the constraints, MCA is not effective for either angle and RMCA shows excellent performance for $\Theta=0.1^{\circ}$. However, for a wider range of look angles, RMCA needs a proper number of low-return constraints to achieve good performance. In general, the performance of RMCA tends to improve as the number of constraints increases from zero, and the number of constraints needs to exceed the number of phase errors to achieve best performance. However, a larger number of constraints are not always beneficial. Normalised root-mean-squared error (NRMSE) also can be a good indicator of performance in simulations, although we cannot compute NRMSE in real situations because we do not have knowledge of the perfectly focused image. The restorations corresponding to the smallest NRMSE are marked with squares.

\subsection{Remarks}

Hence we are considering the challenging case with an i.i.d. phase error, which can occur in practice, we have not presented autofocus examples in this paper using the well-known PGA algorithm. For the i.i.d. phase error case, PGA often does not produce properly focused images for typical data collection angles
[17]. However, for smoother phase errors PGA can be a very good choice.

We presented simulation results and discussed the quality of MCA restorations for an attenuation function modelled by a 2D separable sinc. We also have produced simulation results for other antenna footprints. MCA and RMCA can be more effective in situations where the attenuation function has a steeper fall-off or where the attenuation function is weighted with a tapering function, such as Hamming window to suppress the signal outside the main lobe. The extreme case is when the attenuation is modelled by a rect function, in which case the performance can be nearly perfect, even for MCA when the range of look angles is small.

As we presented in [18], the geometry of the low-return region also influences the performance. The effect of the geometry is not completely understood, but certain guidelines for choosing effective low-return constraints are intuitive. For example, zero-return columns in a perfectly-focused image are not useful at all as low-return constraints. This is because the corresponding columns in any image formed by filtering the defocused image with a 1D kernel are zero-valued, and trying to minimise the energy in such a region is useless. Although zero-return columns are not likely to be encountered, there are columns of low returns in some realistic attenuation windows, and they may be ineffective by a similar argument.

The sampling intervals of the Fourier data also affect the performance. When using PFA to form images, we resampled onto a dense Cartesian grid so that the FOV of the formed image is larger than the scene of interest. This was to provide more low-return pixels near the nulls of the sinc in the spatial domain, so that MCA could utilise the near-zero pixels as the low-return constraints. Since the performance of multichannel autofocus algorithms strongly depends on the level of the low-return region, over-sampling in the Fourier domain is a key to achieving better performance. Similarly, the sampling intervals of the polar data grid should be dense enough to provide a decent set of low-return pixels in the inverse-polar domain. In our simulations, the polar-grid sampling intervals were chosen so that the maximum range and cross-range distances between the samples do not

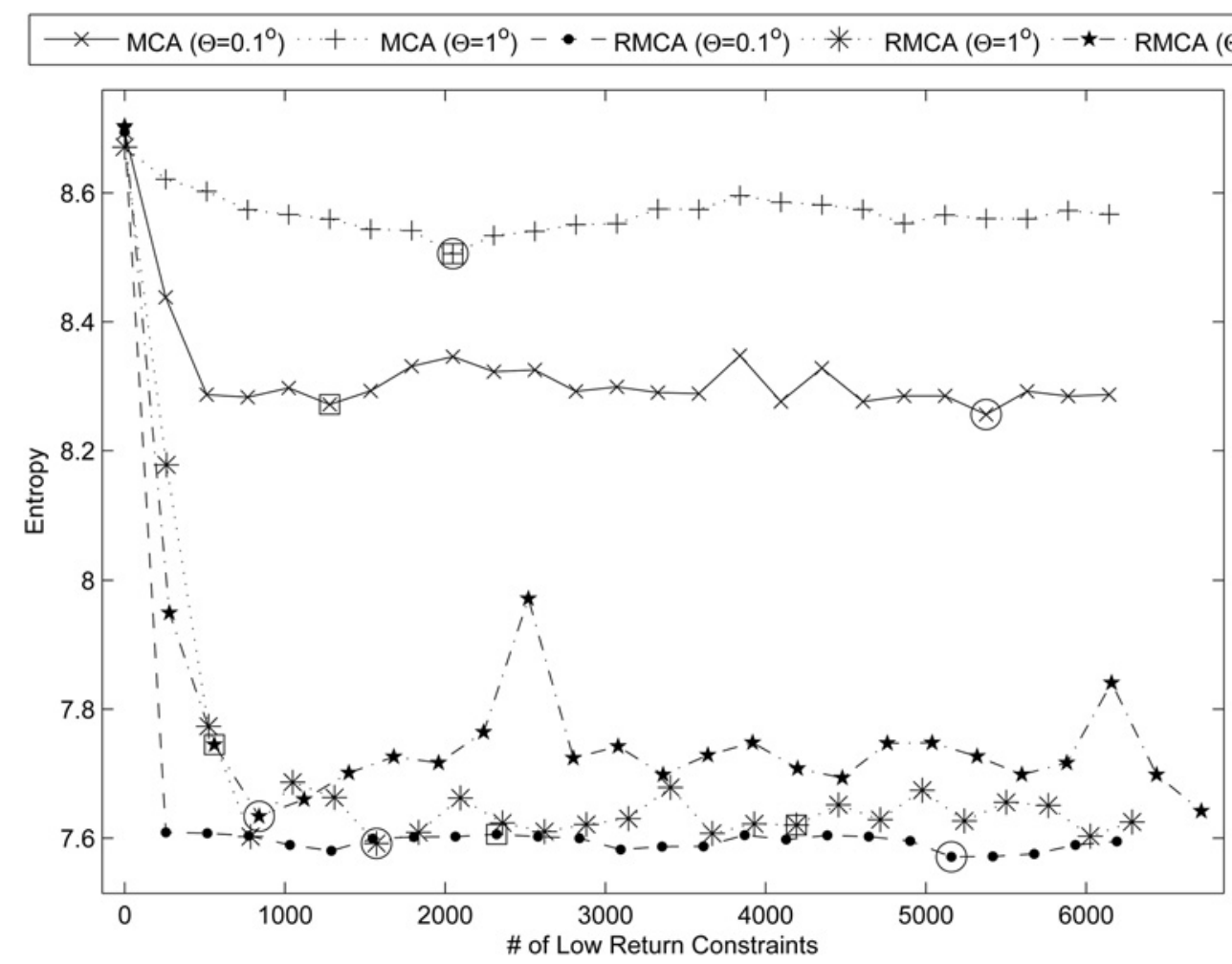

Fig. 7 Performance of the multichannel autofocus algorithms measured by entropy of restored images for various numbers of low-return constraints. The circles indicate the restorations with the lowest entropy measure and the squares indicate the restorations with the smallest NRMSE for each set of simulations 
exceed the range and cross-range sampling intervals of the over-sampled Cartesian grid, respectively. However, the sampling rates were not optimised in any sense, and adequate sampling rates could differ for other antenna footprints. Thus, MCA and RMCA could achieve better performance than presented here if we had used denser Fourier data grids, and more importantly, they will not achieve the quality presented if the Fourier data grids are not sufficiently dense.

The reader might assume that a wider range of look angles in our simulations corresponds to more angular samples and higher-resolution images; however, this is not the case. Rather than fixing the radar centre frequency for all simulations, the centre frequency was changed for different ranges of look angles so that the amount of Fourier coverage remained almost the same. The resolution of restored images and the sizes of the Fourier region covered by the Cartesian grids were fixed for all ranges of look angles, and the polar data grids were chosen so that they circumscribed the Cartesian grids. Thus, the polar grid with a small range of look angles was located further away from the origin in Fourier space. We used this formulation for our simulations to avoid possibly misleading results.

Another recently developed multichannel autofocus algorithm, termed Fourier-domain MCA (FMCA) [17] is more successful for a wider range of look angles than RMCA. The performance of RMCA and FMCA is comparable for fairly small ranges of look angles, and the computational cost for FMCA is much higher because of the need for sophisticated interpolation required for the process of estimating the blurring kernel.

\section{Conclusions}

MCA does not provide the expected performance when used with SAR data collected on a polar grid, even for quite narrow data-collection angles. Our proposed method, RMCA, overcomes this problem by forming the solution space in the inverse-polar domain, where the defocusing effect is truly one dimensional. As with MCA, the performance of RMCA depends on the choice of the low-return constraints; thus, the low-return region must be carefully specified in the inverse-polar domain to achieve the best performance. To gain a better understanding of the inverse-polar domain, we provided an analytical formula for the impulse response. Simulation results demonstrated that, with an adequate selection of low-return constraints, RMCA achieves excellent performance for practical ranges of look angles, up to several degrees.

The performance of multichannel autofocus algorithms is degraded when the attenuation pattern has a relatively wide transition band. In fact, the transition is relatively smooth in the inverse-polar domain compared to that in the spatial domain, and even more, it is smoother for a wider range of look angles. Hence, the robustness to non-zero pixels in the presumed low-return region is very important for RMCA. Fortunately, the quality of RMCA restorations can be improved by incorporating sharpness metric optimisation as a regularisation term, as discussed in [8]. This challenge becomes more extreme if there are strong point reflectors in the weakly illuminated region of the radar beam. Since we cannot detect the strong reflectors prior to focusing the image, we have suggested using random subregions of the presumed low-return region [18], or iteratively refining the image and the low-return constraints. Other possible issues that relate to non-ideal low returns include multiplicative noise due to system non-linearities, displacement of moving targets, and error in the inertial measurement unit of the radar.

The performance of RMCA is affected by variables that must be prescribed, such as the geometry of the presumed low-return region and the number of constraints. Methods for optimal selection of these parameters are not known. By seeking answers to some of these open questions, we believe that we can provide an even more robust algorithm that has better performance in challenging scenarios.

\section{Acknowledgments}

The authors thank R. L. Morrison, Jr. for helpful discussions regarding this work.

\section{References}

1 Munson Jr., D.C., O'Brien, J.D., Jenkins, W.K.: 'A tomographic formulation of spotlight-mode synthetic aperture radar', Proc. IEEE, 1983, 71, (8), pp. 917-925

2 Jakowatz, C.V., Wahl, D.E., Eichel, P.H., Ghiglia, D.C., Thompson, P.A.: 'Spotlight-mode synthetic aperture radar: a signal processing approach' (Kluwer Academic, 1996)

3 Eichel, P.H., Ghiglia, D.C., Jakowatz, C.V., Wahl, D.E.: 'Phase-gradient autofocus for SAR phase correction: explanation and demonstration of algorithmic steps' Proc. Digital Signal Processing Workshop, Starved Rock State Park, IL, September 1992, pp. 6.6.1-6.6.2

4 Jakowatz, C.V., Wahl, D.E.: 'Eigenvector method for maximum-likelihood estimation of phase errors in synthetic-aperture-radar imagery', JOSA A, 1993, 10, (12), pp. 2539-2546

5 Kragh, T.J.: 'Monotonic iterative algorithm for minimum-entropy autofocus'. Proc. Adaptive Sensor Array Processing Workshop, MIT Lincoln Laboratory, MA, June 2006

6 Fienup, J.R., Miller, J.J.: 'Aberration correction by maximizing generalized sharpness metrics', JOSA A, 2003, 20, (4), pp. 609-620

$7 \mathrm{Xi}$, L., Guosui, L., Ni, J.: 'Autofocusing of ISAR images based on entropy minimization', IEEE Trans. Aerosp. Electron. Syst., 1999, 35, (4), pp. 1240-1252

8 Morrison Jr., R.L., Do, M.N., Munson Jr., D.C.: 'MCA: A multichannel approach to SAR autofocus', IEEE Trans. Image Process., 2009, 18, (4), pp. 840-853

9 Cho, H.J., Munson Jr., D.C.: 'Overcoming polar-format issues in multichannel SAR autofocus'. Proc. 42nd Asilomar Conf. Signals, Systems, and Computers, Pacific Grove, CA, October 2008, pp. 523-527

10 Stewart, G.W.: 'Perturbation theory for the singular value decomposition', in Vaccaro, R.J. (Ed.): 'SVD and signal processing, II: algorithms, analysis and applications' (Elsevier, 1990), pp. 99-109

11 Hayes, M.H.: 'The reconstruction of a multidimensional sequence from phase or magnitude of its Fourier transform', IEEE Trans. Acoust. Speech Signal Process., 1982, 30, (2), pp. 140-154

12 Munson Jr., D.C., Sanz, J.L.C.: 'Phase-only image reconstruction from offset Fourier data'. Proc. SPIE 556, Int. Conf. Speckle, San Diego, CA, August 1985, pp. $227-235$

13 Hsieh, J.: 'Computed tomography: principles, design, artifacts, and recent advances' (SPIE, 2003)

14 Hsiao, W.-H., Millane, R.P. 'Effects of Fourier-plane amplitude and phase errors on image reconstruction. I. Small amplitude errors', JOSA A, 2007, 24, (10), pp. $3180-3188$

15 Jones, D.S., Kline, M.: 'Asymptotic expansion of multiple integrals and the method of stationary phase', J. Math. Phys., 1958, 37, pp. 1-28

16 'Synthetic Aperture Radar (SAR) Imagery', http://www.sandia.gov/radar/imagery. $\mathrm{html}$, accessed January 2015, Courtesy of Sandia National Laboratories, Airborne ISR

17 Liu, K.-H., Munson Jr., D.C.: 'Fourier-domain multichannel autofocus for synthetic aperture radar', IEEE Trans. Image Process., 2011, 20, (12), pp. $3544-3552$

18 Cho, H.J., Munson Jr., D.C.: 'Multichannel SAR autofocus using multiple low-return constraints'. Proc. IEEE Int. Conf. Acoustic, Speech, and Signal Processing, Dallas, TX, March 2010, pp. 1346-1349 